Schon in meiner Doktorarbeit habe ich darauf aufmerksam gemacht, daß das wahre Trägheitsmoment infolge der magnetischen Energie vergrößert wird.

Nach den Gleichungen (94) und (89) gilt

$$
\dot{\mathrm{M}}=\frac{2}{\delta^{4}}\left[\frac{1}{\delta} \frac{\operatorname{Sin} \delta-\sin \delta}{\operatorname{So} \delta-\cos \delta}+2 \frac{\operatorname{Co} \delta \delta-\cos \delta-1}{(\operatorname{Co} \delta-\cos \delta)^{2}}\right] .
$$

Für kleine Geschwindigkeiten ergibt sich

$$
\mathrm{M}=4\left[\mathrm{I} \frac{\mathrm{s}_{6}}{\pi^{6}}-2 \frac{\mathrm{s}_{10}}{\pi^{10}} \gamma^{2}+3 \frac{\mathrm{s}_{14}}{\pi^{14}} \gamma^{4}-\ldots\right]
$$

Ist $\gamma$ sehr klein, können wir uns auf

$$
\mathrm{M}=4,232 \cdot 10^{-3}
$$

beschränken, so daß nach (94) für Kupfer folgt

$$
\mathrm{J}^{\prime}=\mathrm{I}, 637 \cdot \mathrm{IO}^{-7} \mathrm{H}_{\mathbf{0}}{ }^{2} \mathrm{a}^{7} \text {. }
$$

Für großé Geschwindigkeiten verschwindet $M$ gemäß der Formel

$$
\mathrm{M}=\frac{\mathrm{I}}{2 \sqrt{2}} \gamma^{-\frac{5}{2}}=0,3535 \gamma^{-\frac{5}{2}}=\frac{2}{d^{5}} \text { : }
$$

Die letzte Kolonne der Tabelle 2 gibt $M$ als Funktion von $\gamma$.und $\delta$, und Bild 6 stellt $M$ in seiner Abhängigkeit von $\gamma$ dar.

7. Schlußbemerkungen. Hiermit haben wir das Problem in aller Strenge behandelt unter der einzigen Voraussetzung, daß sich im Außenraum kein Eisen befindet, durch welches der Kraftlinienverlauf deformiert würde. Unsere Formeln gelten also unter der Annahme, daß das statische Magnetfeld durch elektrische Ströme hervorgerufen wird, oder daß die Eisenmassen der Elektromagnete sich in so großer Entfernung von der Kugel befinden, daß sie das Feld der induzierten Strome nicht mehr wesentlich beeinflussen können.

Die eben erwähnte Deformation der Kraftlinien durch die Eisenmassen der Elektromagnetkerne scheint mir die größte Schwierigkeit für eine strenge Theorie der Wirbelstrombremsen zu bilden. Ich werde auf diesen Punkt demnächst zurück. kommen, wenn, ich eine Anordnung behandle, die sich der Praxis wesentlich mehr nähert als das oben gelöste Problem. Indessen glaube ich doch, daß letzteres nicht ohne Wert ist, weil es alle Phänomene zum Ausdrück bringt, die man an elektromagnetischen Bremsen tatsächlich beobachtet, und weil es erwünscht schien, an einem wenn auch etwas idealisierten Beispiel mittels der Maxwell-Hertzschen Theorie einmal in Strenge alle Einzelheiten quantitativ durchzurechnen.

La Plata, 28. Juni 1919.

Instituto de Física.

\title{
Die stationären Schwingungen der wechselstromgespeisten Spule.
}

\section{Berichtigung.}

Die Unterschriften der Bilder Io und II meiner Arbeit (Seite 353 dieses Bandes) enthalten leider eine Verwechselung. Es bedeutet in beiden Spektren 